\title{
Lost and Found: A Case of Continued Migration of an IUD Allowing for Removal via Cystoscopy
}

Alissa Dangel, Katherine Hicks-Courant, Tony Luongo and Tanaz R. Ferzandi ${ }^{*}$

Departments of Obstetrics and Gynecology and Urology, Tufts Medical Center, 800 Washington St, Boston, MA 02111, USA

\section{Abstract}

Uterine perforation is a known complication of intrauterine device (IUD) insertion. As long-acting reversible contraception use increases, the frequency of uterine perforation at the time of IUD insertion will also rise. In rare cases, the IUD can also perforate the bladder or the bowel. In this case report, we present an incidental finding of an echogenic focus on a routine nuchal translucency ultrasound, leading to the discovery of a "lost" IUD.

\section{Publication History:}

Received: March 17, 2016

Accepted: October 17, 2016

Published: October 19, 2016

\section{Keywords:}

Cystoscopy, Intrauterine device, Contraception, Pregnancies

\section{Case Presentation}

A 28 year-old G1P0 at 9 weeks of gestation presented for prenatal care in 2010. She was sent for a routine nuchal translucency ultrasound, during which an echogenic focus was noted abutting the bladder The dimensions were consistent with an intrauterine device (IUD) (Figure 1). The patient's history was significant for a copper IUD that had been inserted in 2003. Following the insertion, the patient had episodes of pelvic pain. She then presented for a physical exam in 2004 at which time the strings were not visualized in the cervix. An ultrasound was ordered and when the IUD was not identified, it was presumed expelled.

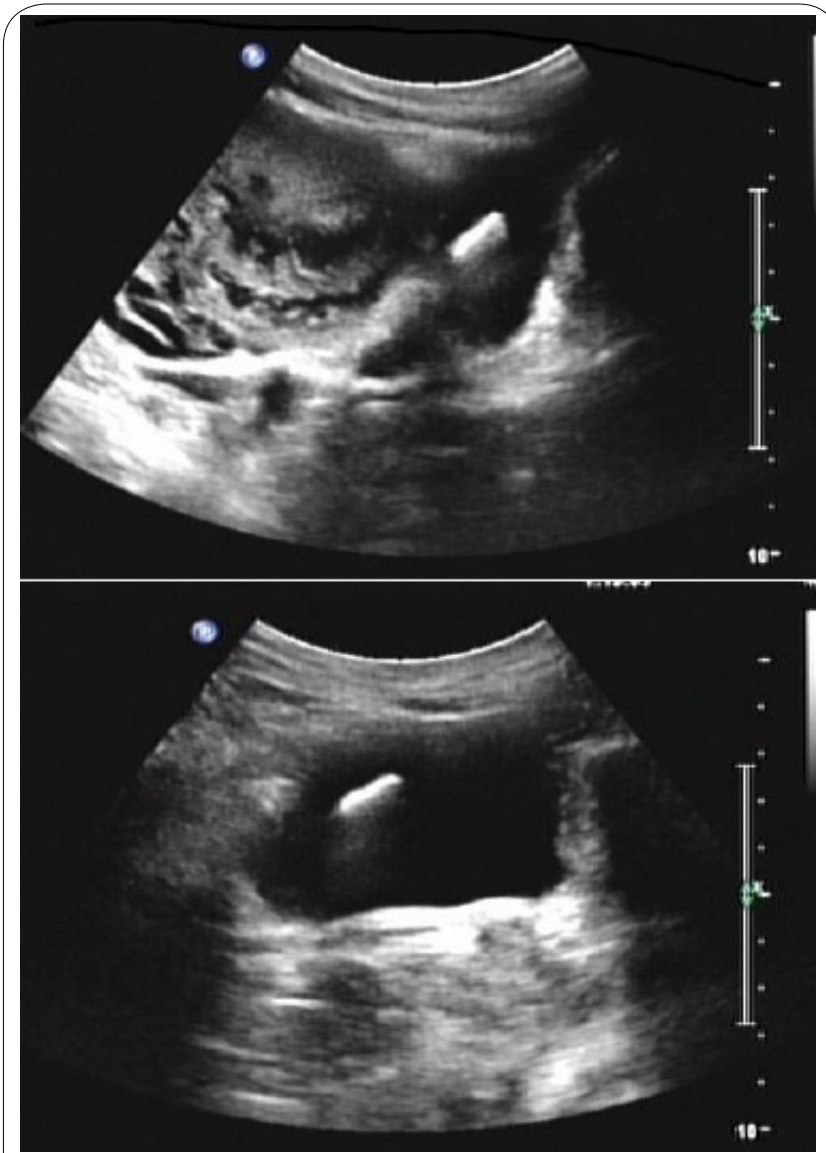

Figure 1: Consistent with an intrauterine device.
After the ultrasound concerning for an IUD abutting the bladder, the patient was referred to a Urogynecologist where she underwent a cystoscopy. The cystoscopy confirmed the presence of the IUD in the base of the bladder (Figure 2). The patient subsequently had a spontaneous vaginal delivery of this pregnancy followed by three

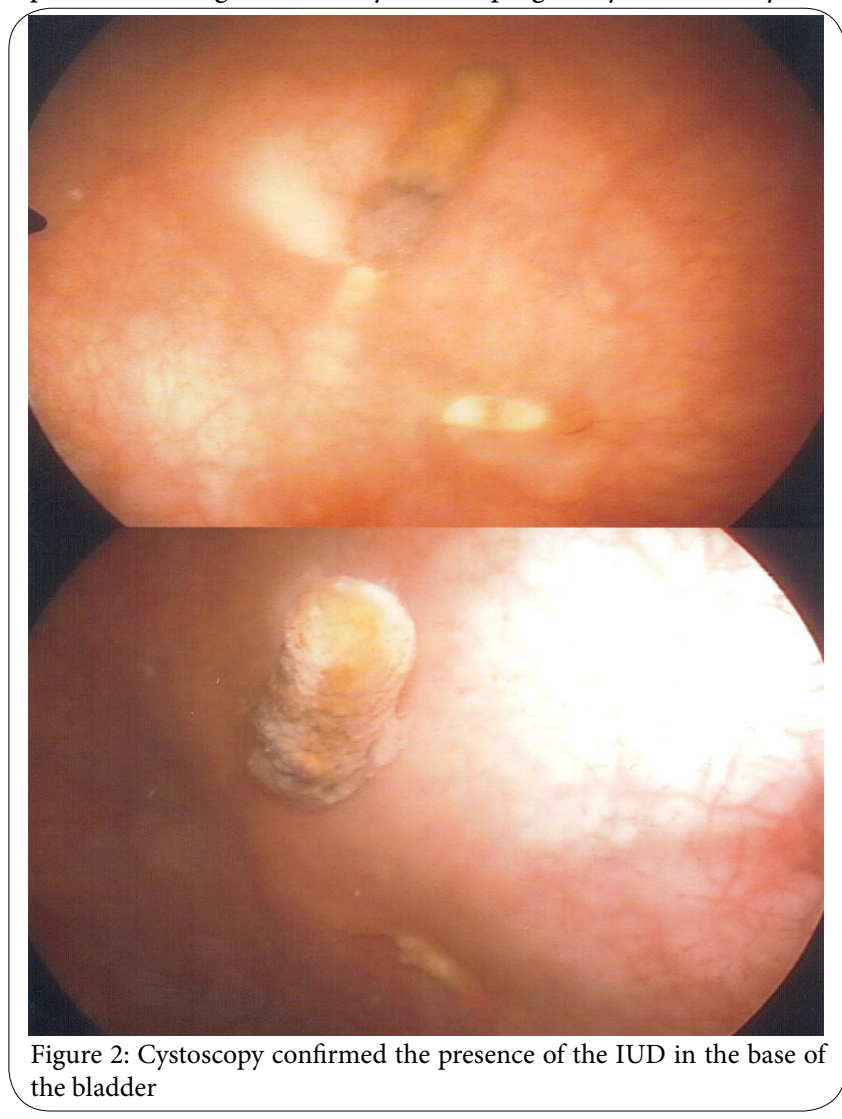

"Corresponding Author: Dr. Tanaz R. Ferzandi, Departments of Obstetrics and Gynecology and Urology, Tufts Medical Center, 800 Washington St, Boston, MA 02111, USA; E-mail: tferzandi@tuftsmedicalcenter.org

Citation: Dangel A, Courant KH, Luongo T, Ferzandi TR (2016) Lost and Found: A Case of Continued Migration of an IUD Allowing for Removal via Cystoscopy. Int J Gynecol Clin Pract 3: 123. doi: http://dx.doi.org/10.15344/2394-4986/2016/123

Copyright: (c) 2016 Dangel et al. This is an open-access article distributed under the terms of the Creative Commons Attribution License, which permits unrestricted use, distribution, and reproduction in any medium, provided the original author and source are credited. 
additional pregnancies over the course of approximately two years. These resulted in a miscarriage treated with dilation and curettage complicated by hemorrhage, a second vaginal delivery, and a spontaneous miscarriage. The patient then presented for surgical removal of the IUD and for permanent sterilization. She underwent cystoscopic removal of the IUD, which by that time had almost entirely migrated into the bladder cavity (Figure 3). Laparoscopy was performed to repair the cystotomy that was created during the extraction and the bladder defect was sutured. A simultaneous laparoscopic tubal ligation was performed.

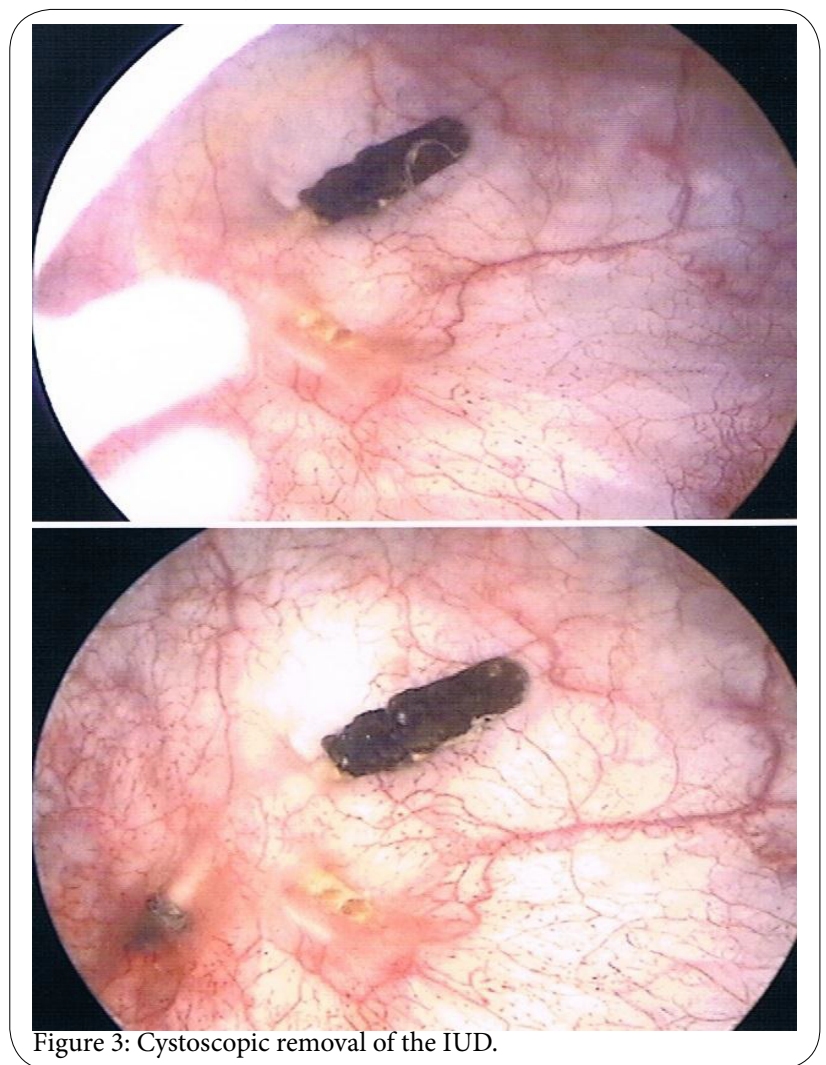

\section{Discussion}

Uterine perforation is a known complication of IUD insertion with a rate of approximately 1.2 per 1,000 insertions, with the bladder being a particularly rare site [1]. This case illustrates that an extrauterine IUD continued to migrate even after the initial perforation. It is likely that this migration occurred as a result of the patient's subsequent pregnancies (as the uterus would have been enlarging), or it could be a physiologic process following uterine perforation. In this case, the subsequent pregnancies and resultant delay of definitive surgery may have allowed for continued migration of the device, ultimately allowing for removal by cystoscopic resection and extraction, rather than an abdominal approach. This case raises the question of consideration of expectant management of a perforated IUD in the hopes of retrieving it via a less invasive method depending on its location and the clinical symptoms of the patient.

\section{References}

1. Honarbakhsh A, Rastegar M, Heravi I, Khoury E. (2003) Unrecognised perforation of the uterus into bladder by an IUD. J Obstet Gynaecol 23: 444-445. 